\title{
Effects of anxiety on dominant and nondominant intratask responses: A modified replication'
}

\author{
MARTIN KATAHN AND SALLY T, KOPLIN \\ VANDERBILT UNIVERSITY
}

Modifications of a previous study (Bemstein, 1963) which had failed to illustrate the facilitating effects of anxiety on the acquisition of dominant intratask responses and the interfering effects of anxiety on nondominant responses led to findings which generally support predictions from drive (anxiety) theory. Possible defects in the earlier design are discussed.

Pursuing a suggestion by Hill (1957), two studies (Katahn, 1964; Katahn \& Lyda, 1966) have investigated the effects of anxiety in a verbal learning task in which it is possible to demonstrate the relative strength of four intratask response classes for each $\mathrm{S}$ prior to learning. High anxiety was found to facilitate the acquisition of responses in the dominant class and to interfere with the acquisition of responses in the lower ranking response classes. These results supported predictions derived from Spence-Taylor drive theory (Spence, 1958).

Bernstein (1963) also devised a technique for determining the relative strength of two intratask response tendencies in a variant of a serial verbal maze using nonsense syllables (CVCs) as choice points. His results, however, clearly failed to support predictions from drive theory. As described in the method section below, the present study sought to remedy three possible defects in Bernstein's design; (1) all CVCs used in the original study were of equal association value (47\%) according to Glaze, which might have contributed to a lack of discriminability at each choice point, (2) a "within" Ss design was employed wherein each S was reinforced half the time for responding with his dominant response tendency and half the time for responding with the nondominant response tendency, thus permitting generalization and intratask competition amongst CVCs of low discriminability, that is, syllables of similar association value were sometimes right and sometimes wrong for each S, and (3) reinforcement consisted of the word "right" for correct responses, which may not provide enough motivation to maximize the selection of correct responses for curious college student Ss. Method

Twenty-eight male and 28 female Ss with scores in the upper and lower 25\% of the distribution of Taylor Anxiety Scale scores for each sex served in the experiment as part of their requirements in an introductory psychology course. High anxiety (HA) Ss had scores ranging from $21-38$, and low anxiety (LA) Ss had scores ranging from 1-9. A twelve unit verbal maze was constructed using 12 high and 12 low pronounceable (Pr)
CVCs obtained from Underwood \& Schulz (1960). Hard to pronounce items had scale values from 5.04 to 7.52 , and a range of Glaze association values from 0-33; easily pronounced items had scale values ranging from 1.99 to 2.92 , and Glaze association values between 60100. Each pair of items in the maze consisted of one high and one low Pr CVC, randomly selected. The position of the high and the low Pr CVC as the left or right member of the pair was also randomized.

Each pair of items in the maze was exposed for $4 \mathrm{sec}$. on a Lafayette model 303B memory drum. There was no delay between presentations nor any intertrial interval. A recorded buzzer sound of approximately $100 \mathrm{~dB}$ was administered manually through headphones for approximately .5 sec. for wrong responses.

The $S$ was told that he would see in the window of the drum a series of nonsense syllable pairs, one of which was arbitrarily selected by the E as "correct." In accord with Bernstein's suggestions, the first trial was a free choice (FC) trial. On this trial the $S$ was told that $E$ would not indicate which response was actually right but $S$ was to select and spell out which ever one he thought might be correct after looking at both. A $60 \mathrm{sec}$. interval separated the FC trial from learning Part 1 (L1).

Following the FC trial Ss were again told to spell the item that they thought was correct, but that from now on the buzzer would sound whenever they gave an incorrect response. They were told that their job was to learn all the correct syllables and that criterion was two consecutive trials of complete buzzer avoidance. In contrast with Bernstein's L1 procedure in which each $S$ was reinforced an equal number of times for giving dominant and nondominant responses, the present study employed a between-Ss design. Half the Ss of each sex in each anxiety group were reinforced for repeating all their initial (dominant) responses and the other half were reinforced for choosing syllables not selected on the FC trial (nondominant CVCs).

After reaching criterion on $\mathrm{L} 1$ Ss received another $60 \mathrm{sec}$. break. Learning Part 2 (L2) followed in which all Ss were obliged to switch to the response which had been incorrect during $\mathrm{L} 1$. Thus, in L2, initial dominance was ignored, and all Ss were obliged to extinguish the response which had acquired dominance through reinforcement during L1. A criterion of two errorless trials was again established.

\section{Results and Discussion}

As would be predicted from Spence-Taylor theory, HA Ss reinforced for giving initially dominant responses 
averaged the fewest trials (3.64) to reach criterion, while HA Ss reinforced for giving nondominant responses averaged the greatest number of trials (6.71). The interaction, Anxiety by Dominance, was significant $(F=4.77, d f=1 / 48, p<.05)$. LA Ss reinforced for initially dominant and nondominant responses fell in between the extremes established by the HA groups. LA Ss in the initially dominant and nondominant groups averaged 6.57 and 5.64 trials to criterion, respectively. The reversal in effects for LA Ss in the dominant and nondominant conditions appeared to result from the performance of low anxious males, although no significant interactions with sex were obtained. Low anxious males were the slowest learning group over all, especially those in the dominant condition. This group averaged 8.75 trials to reach criterion. Some unusual nonrepetitive factor appears to have been operating during L1 for the low anxious male dominant group since this group did not prove to be the slowest group during L2.

In reversal learning on L2, HA Ss now being reinforced for giving responses of low acquired dominance took longer to reach criterion than did LA Ss, 4.96 trials to 4.68 trials. While this was far from significant $(F<1)$, it is in the direction predicted from drive theory. Certain characteristics of the task may have minimized the difference between HA and LA groups during L2. The second part of the task proved generally easier than the first part (grand mean L1 $=5.64$ trials, grand mean L2 $=4.82$ trials). The decrease in difficulty may be due to the use of only two intratask response classes. Reversal learning simply required Ss to give the previously avoided response in all cases after having well learned the nature and relationship of each CVC pair during L1. Thus, the Bernstein type of experimental task may not be as adequate a situation for demonstrating the interfering effects of anxiety on lower ranking intratask responses as is a task con- taining four response classes, each class in turn containing twice the number of members as were contained in the present experiment (Katahn \& Lyda, 1966). Ss in the Katahn and Lyda experiment took significantly longer to reach criterion on the lower ranking response classes than they did on the dominant class, after having been reinforced for giving responses on the dominant class.

The results of the present study and the other studies referred to in this paper highlight the difficulties of explicating drive theory. The variables investigated affect so little of the variance in complex human learning that careful control of task factors as suggested by Hill (1957), not to mention a multitude of other subject variables, becomes essential for an adequate test of the theory. The present modifications in Bernstein's procedures, and the other studies mentioned above, suggest that Bernstein's original experimental design was not quite adequate for such a test.

\section{References}

Bemstein, A. S. Anxiety as a nondirectional drive: A test of HullSpence theory. Psychol. Rep., 1963, 12, 87-98.

Hill, W. F. Comments on Taylor's "drive theory and manifest anxiety." Psychol. Bull., 1957, 54, 490-493.

Katahn, M. Effects of anxiety (drive) on the acquisition and avoidance of a dominant intratask response. J. Pers., 1964, 32, 642650.

Katahn, M., \& Lyda, L. L. Anxiety and the learning of responses varying in initial rank in the response hierarchy. J. Pers., 1966 34, 287-299.

Spence, $\mathbf{K}$, W. A theory of emotionally based drive (D) and its relation to performance in simple learning situations. Amer. Psychologist, 1958, 13, 131-141.

Underwood, B. J., \& Schulz, R. W. Meaningfulness and verbal learning. New York: J. B. Lippincott, 1960.

\section{Note}

1. This research was supported by a grant $\mathrm{MH}-11149$ from the $\mathrm{Na}$ tional Institute of Health of the United States Public Health Service. 